\title{
Modeling and Simulation of a Linear Piezoelectric Stepper Motor in MapleSim
}

\author{
Orysia Soroka \\ Derek Wright \\ Orang Vahid \\ Maplesoft \\ Waterloo, Ontario, Canada \\ ooasorok@uwaterloo.ca,dwright@maplesoft.com, ovahid@maplesoft.com
}

\begin{abstract}
Devices based on piezoelectric materials have traditionally been modeled in PDE simulation software. These simulations are expensive to create and run. In this paper it is shown that lumped-parameter models of such devices can provide good fidelity with low computational cost. Modelica models of supporting components, along with a system-level model of a linear piezoelectric stepper motor are presented. The simulation results show good agreement with published experimental results. Future research is proposed based on the components and model.
\end{abstract}

Keywords: Piezoelectric, Linear Motor, MapleSim

\section{Introduction}

Piezoelectric materials experience mechanical stress under the influence of an electric field and, inversely, produce an electric field with the application of a mechanical stress. Materials that exhibit the piezoelectric effect are used in diverse applications, including a variety of sensors and actuators, and specifically in stepper motors. Detailed PDE simulations of these materials are achievable using simulation software such as COMSOL, but lumped-parameter models suitable for component- and system-level simulations are rare. Developing piezoelectric materials models in Modelica makes modeling and simulation at the system-level possible. A resulting library of parametrically-defined component models, like motors and actuators, would increase the efficiency of modeling and simulating piezoelectric devices routinely deployed in new engineering designs.

In this research, Modelica components implementing piezoelectric material properties, electrostatic forces, and time-varying frictions were developed and integrated into a device-level model of a linear piezoelectric stepper motor. The model is parametric and extensible: the parameters can be changed to suit application-specific requirements, and nonlinear effects can be easily included.

MapleSim is a Modelica-based system-level modeling and simulation platform provided by Maplesoft [1]. MapleSim simulation results matched those in [2] when similar values were implemented. Most importantly, the relative execution speed of the model permits multi-parameter optimizations not possible in full PDE simulations. This is demonstrated via the investigation of the effects of the motor clamp voltage on velocity using a compiled MapleSim procedure in Maple. Future work is then described.

\subsection{Related Work}

To the authors' knowledge, there is no formal Modelica library available for piezoelectric materials. However, there have been a variety of disparate works that have implemented piezoelectric models in a lumped-parameter framework. For example, a MEMS library and airbag deployment example including piezoelectric elements were implemented in VHDL-AMS in [3] and [4], respectively. Lumpedparameter models of piezoelectric devices derived from high-order FEM models, are presented in [5], but are not Modelica-specific implementations. They would retain some of the discretized nature of the original FEM models and would therefore be further away from the benefits of using Modelica. In [6], bond graph and equivalent circuit methods are used to model piezoelectric motors. Finally, several toolindependent lumped-parameter physics-based models are presented in Chapter 6 of [7].

\section{Linear Motor Operation}

Figure 1 shows the configuration and operation of the linear motor. The operation is similar to other slip-stick motors, but is unique in that an electrostatic clamp is used to aid the "stick" portion of the cy- 
cle. Periodic waveforms are applied to extend and relax the piezoelectric material along its longitudinal direction, pushing the lead weight along with it. The electrostatic clamp is active during the extension part of the cycle to prevent the motor assembly from slipping along the surface. An abrupt voltage is applied to the piezoelectric material when it is in its extended state and the clamp is deactivated to cause the assembly to retract towards its new center of mass, moving it forward.

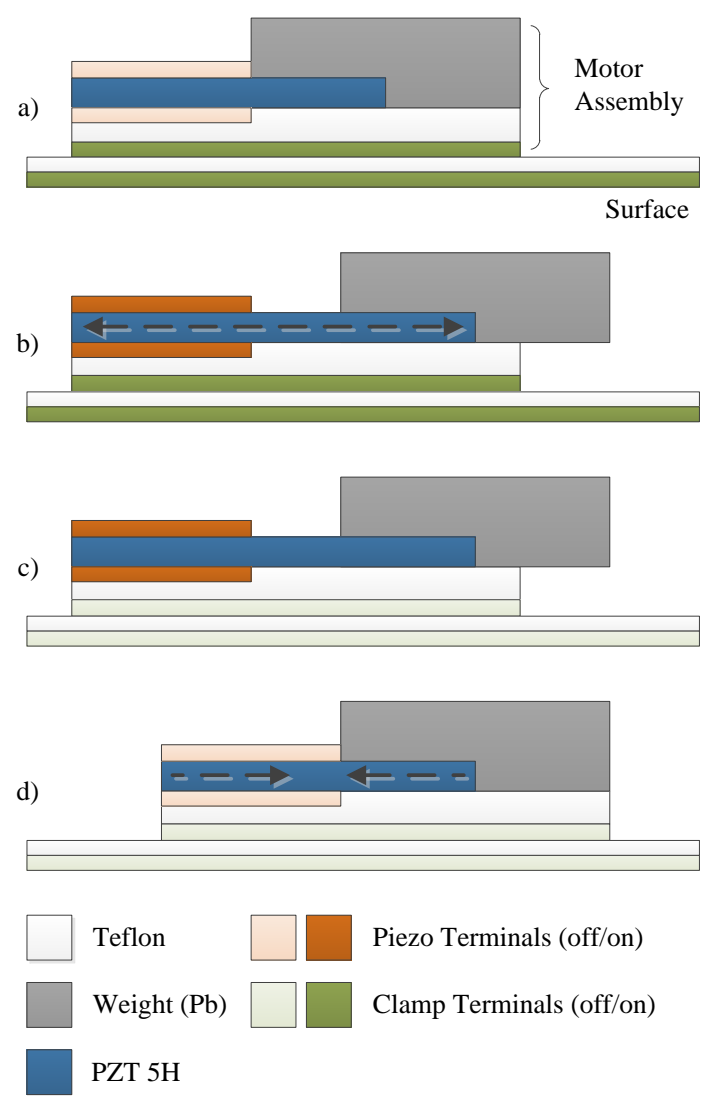

Figure 1: Linear motor configuration and operation. a) The electrostatic clamp is activated. b) The piezoelectric material extends longitudinally with an applied voltage, moving the center of mass to the right. c) The clamp is deactivated. d) The piezoelectric voltage is quickly removed to cause a snapping motion, breaking the static friction between the motor assembly and the surface. The assembly retracts towards its new center of gravity, moving forward.

To model this in MapleSim via Modelica, several new components were needed: A 1D model of the piezoelectric material which couples the electrical and translational domains, an electrostatic clamp that also couples the electrical and translational domains, and a time-varying friction model. Their development is described next.

\section{Component Models}

In the following sections, variables indicated in bold face correspond to port variables. Numbers in brackets preceding an equation (like (1), for example) indicate equations that appear in the final Modelica component.

These components were first created as MapleSim Custom Components, which directly implement their governing equations developed in Maple. Essentially, the equations are written unsimplified and MapleSim automatically rearranges and manipulates them as needed. Upon creation of the component, Modelica code is auto-generated which was then manually further modified.

\subsection{Piezoelectric Material Model}

The development of a 1D piezoelectric model relied heavily on Chapter 6 of [7]. The full tensor solution was reduced to the $(3,3)$ direction to select the longitudinal translational mode of operation. Losses and nonlinearities, such as hysteresis, were neglected as a first-order approximation. Such effects can be easily included in the core equations, or included externally using Modelica Standard Library components. Maxwell's equations were accordingly simplified. Refer to Figure 2 for referencing of the port variables.

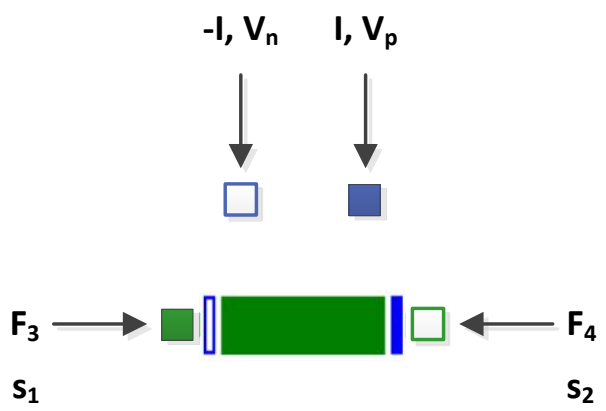

Figure 2: Through- and across-variable references for the piezoelectric component.

In one dimension, the traction (stress) of a piezoelectric material is

$$
T=c^{D} \cdot S-h \cdot D
$$

where $T$ is the traction, $c^{D}$ is the mechanical stiffness of the material, $S$ is the mechanical strain, $h$ is a piezoelectric coupling coefficient with units of $\mathrm{V} / \mathrm{m}$, and $D$ is the electrical displacement field. Neglecting inertia, the forces at either end of a slab of length $l$ and area $A$ of this material are

$$
F_{1}=-\left.A \cdot T\right|_{z=0}, F_{2}=-\left.A \cdot T\right|_{z=l}
$$

noting that traction is referenced positive in the tensile direction. Therefore

$$
F_{1}=-\left.A\left(c^{D} \cdot S-h \cdot D\right)\right|_{z=0}
$$


The strain can be approximated by taking the first derivative of the material's displacement in Eulerian coordinates, $\xi$, so that

$$
S \cong \frac{\partial \xi}{\partial z}
$$

and therefore

$$
F_{1}=A\left(h \cdot D-\left.c^{D} \cdot \frac{\partial \xi}{\partial z}\right|_{z=0}\right)
$$

The $D$ field can be replaced with the charge, $Q$, as follows:

$$
\begin{aligned}
& \frac{\partial D}{\partial t}=J_{\text {disp }} \\
& \oint J_{\text {disp }} \cdot d A=\boldsymbol{I} \\
& \therefore J_{\text {disp }}=\frac{\boldsymbol{I}}{A} \\
& \therefore \frac{\partial D}{\partial t}=\frac{\boldsymbol{I}}{A}
\end{aligned}
$$

Since

$$
\begin{aligned}
& \text { (1) } \boldsymbol{I}=\frac{d Q}{d t} \\
& \therefore D=\int \frac{\boldsymbol{I}}{A} \cdot d t=\frac{Q}{A} \\
& F_{1}=h \cdot Q-\left.A \cdot c^{D} \cdot \frac{\partial \xi}{\partial z}\right|_{z=0}
\end{aligned}
$$

where $J_{\text {disp }}$ is the displacement current and $\boldsymbol{I}$ is the electrical current. Noting that

$$
\begin{aligned}
& \left.\frac{\partial \xi}{\partial z}\right|_{z=0} \cong \frac{\xi_{2}-\xi_{1}}{l}=d s \\
& (\mathbf{2}) d s=\boldsymbol{s}_{\mathbf{2}}-\boldsymbol{s}_{\mathbf{1}}-l
\end{aligned}
$$

Therefore,

$$
\begin{aligned}
& \text { (3) } F_{1} \cong h \cdot Q-A \cdot c^{D} \cdot \frac{d s}{l} \\
& \text { (4) } F_{2}=-F_{1}
\end{aligned}
$$

To incorporate inertia, one-half of the calculated mass is placed on either side of the piezoelectric material. It is calculated from its density, $\rho$, length and area. Damping could also be included in these equations, but was not necessary for this particular analysis.

$$
\begin{aligned}
& \text { (5) } \boldsymbol{F}_{\mathbf{3}}-F_{1}=\frac{1}{2} \rho \cdot l \cdot A \cdot \frac{\partial^{2} \boldsymbol{s}_{\mathbf{1}}}{\partial t^{2}} \\
& \text { (6) } \boldsymbol{F}_{\mathbf{4}}-F_{2}=\frac{1}{2} \rho \cdot l \cdot A \cdot \frac{\partial^{2} \boldsymbol{s}_{\mathbf{2}}}{\partial t^{2}}
\end{aligned}
$$

Finally, the terminal voltage, $\boldsymbol{V}$, can be calculated as the integral of the electrical field, $\mathcal{E}$, as

$$
\boldsymbol{V}=\int_{0}^{l} \varepsilon \cdot d z
$$

Since $\mathcal{E}$ can be defined as a function of $D$ and $S$ via

$$
D=e \cdot S+\varepsilon^{S} \cdot \mathcal{E}
$$

where $e$ is the $(3,3)$ element of the piezoelectric stress matrix. It can be defined as

$$
e=\varepsilon^{S} \cdot h
$$

where $\varepsilon^{S}$ is the electrical permittivity of the piezoelectric material under constant strain conditions. Therefore,

and

$$
\mathcal{E}=\frac{D}{\varepsilon^{S}}-h \cdot S \cong \frac{Q}{A \cdot \varepsilon^{S}}-h \cdot \frac{\partial \xi}{\partial z}
$$

$$
\begin{aligned}
& V=\int_{0}^{l}\left(\frac{Q}{A \cdot \varepsilon^{S}}-h \cdot \frac{\partial \xi}{\partial z}\right) \cdot d z \\
& \text { (7) } V=\frac{Q \cdot l}{A \cdot \varepsilon^{S}}-h \cdot d s
\end{aligned}
$$

where

$$
\text { (8) } V=\boldsymbol{V}_{\boldsymbol{p}}-\boldsymbol{V}_{\boldsymbol{n}}
$$

\subsection{Electrostatic Force Model}

An electrostatic force model was implemented that couples the electrical and translational domains. Unlike in the piezoelectric model which did incorporate a linear stress-strain relationship, the stress-strain relationship of the dielectric material under the influence of the applied electrostatic force was not included. It is present in the system-level model as a translational spring. This decision was made so that the component could be easily modified as needed. For example, more accurate models would use a translational spring-damper to incorporate losses, and keeping it outside the electrostatic force component facilitates this change. Refer to Figure 3 for referencing of the port variables.

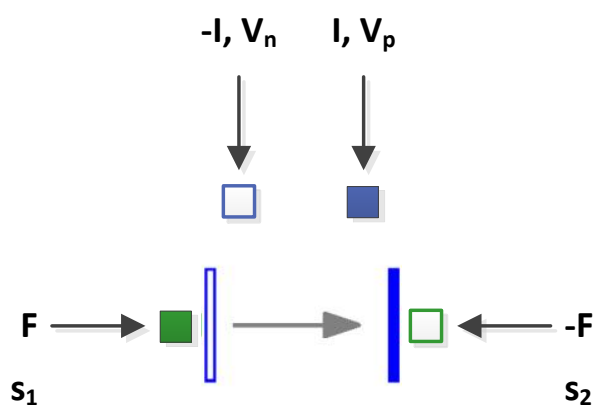

Figure 3: Through- and across-variable references for the electrostatic force component.

Neglecting edge effects, the force between two plates of a parallel capacitor and current are

where
(1) $\boldsymbol{F}=\frac{Q \cdot V}{d}=\frac{C \cdot V^{2}}{d}$
(2) $C=\frac{\varepsilon_{0} \cdot \varepsilon_{r} \cdot A}{d}$
(3) $I=\frac{d}{d t}(C \cdot V)$

and

(4) $d=\boldsymbol{s}_{2}-\boldsymbol{s}_{1}$

(5) $V=V_{p}-V_{n}$. 


\subsection{Smooth Time-Varying Friction Model}

The purpose of this model was twofold: First, a timevarying friction was needed where the normal force and coefficients were time-dependent. This was due to the electrostatic clamp changing the applied normal force. Second, whereas the standard friction model is discontinuous when transitioning from static to dynamic, a continuous model would produce similar results and would speed simulation time by avoiding events. It also eliminated the need to provide scaling information to the solver to detect events within such a narrow band of operation. Refer to Figure 4 for referencing of the port variables.

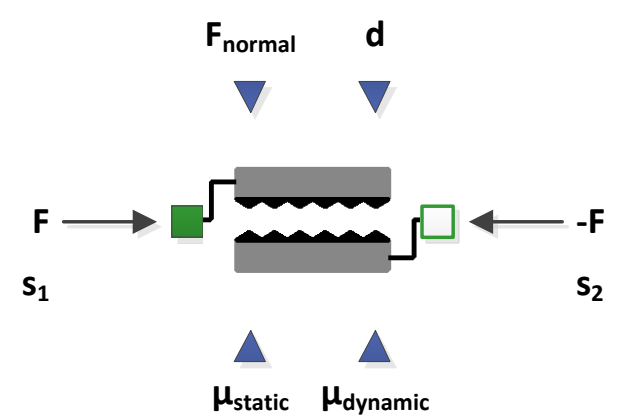

Figure 4: Through- and across-variable references and input signals for the time-varying friction component.

Beginning with the smooth friction model, a sum of two hyperbolic tangents was used to create the approximation.

(1) $g\left(x, A_{1}, A_{2}\right)=A_{1} \cdot \tanh \left(c_{1} \cdot x\right)$

$$
+\left(A_{2}-A_{1}\right) \cdot \tanh \left(c_{2} \cdot x\right)
$$

In its intended usage, $x$ would be the relative velocity, $A_{1}$ would be the static friction, and $\left(A_{2}-A_{1}\right)$ would be the dynamic friction. The coefficients $c_{1}$ and $c_{2}$ are chosen so that $c_{1}>c_{2}$, which gives the desired function shape. An example is shown in Figure 5 and its similarity to the basic discontinuous friction model should be noted.

Similarly, a smooth step-like function was used to ensure that when non-positive normal force is applied, there is no resultant "negative" friction. Such a function was implemented using

$$
\text { (2) } h(x)=\frac{1}{2}\left(\tanh \left(c_{3} \cdot x\right)+1\right)
$$

and an example plot is shown in Figure 6.

Using these smooth equations, the friction model is then implemented as
(3) $s_{r e l}=\boldsymbol{s}_{\mathbf{2}}-\boldsymbol{s}_{\mathbf{1}}$
(4) $v_{\text {rel }}=\frac{d s_{r e l}}{d t}$
(5) $F_{\text {static }}=\boldsymbol{\mu}_{\text {static }} \cdot \boldsymbol{F}_{\text {normal }} \cdot h\left(\boldsymbol{F}_{\text {normal }}\right)$
(6) $F_{\text {dynamic }}=\boldsymbol{\mu}_{\text {dynamic }} \cdot \boldsymbol{F}_{\text {normal }}$

$$
\begin{gathered}
\cdot \boldsymbol{h}\left(\boldsymbol{F}_{\text {normal }}\right) \\
\mathbf{F}=g\left(v_{\text {Rel }},\right. \\
\left.F_{\text {static }}, F_{\text {dynamic }}-F_{\text {static }}\right) \\
+\boldsymbol{d} \cdot\left(v_{\text {Rel }}\right)
\end{gathered}
$$

where $\boldsymbol{d}$ is the damping coefficient.

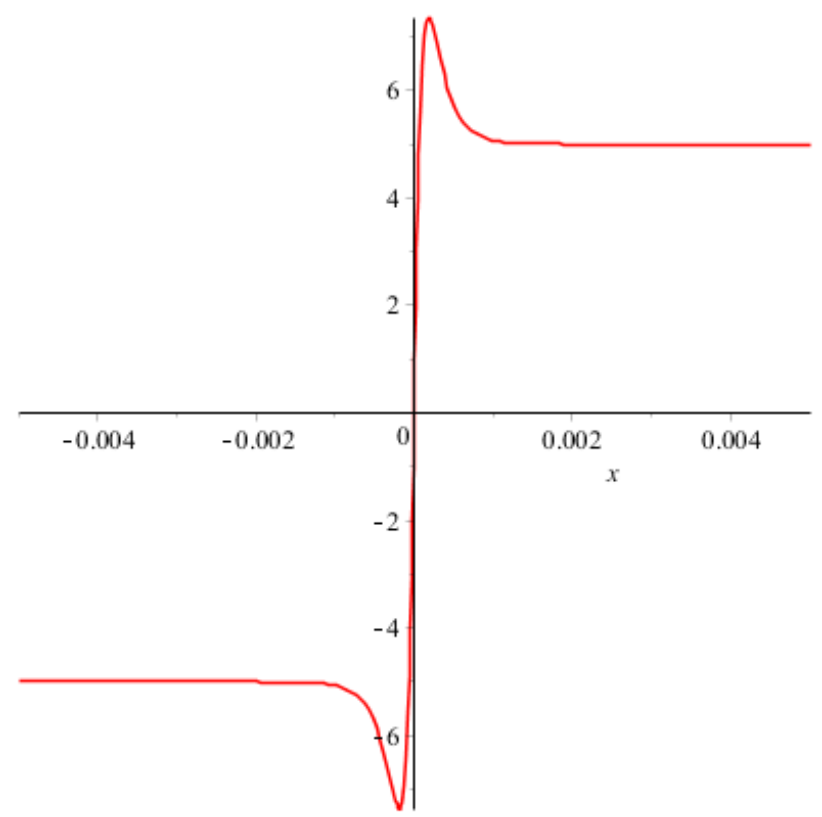

Figure 5: Example plot of the smooth friction model for parameters: $A_{1}=10, \quad A_{2}=5, \quad c_{1}=10000$, $c_{2}=2500$.

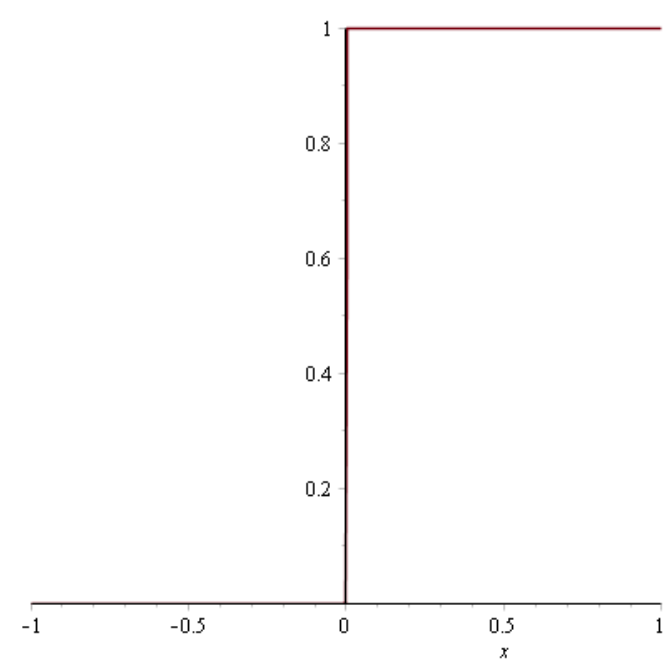

Figure 6: Example plot of the smooth step function for the parameter $c_{3}=10000$.

\section{Slip-Stick Motor Model}

The three new Modelica components were assembled in MapleSim along with library 1D translational and signal components to create the overall model, shown in Figure 7. 


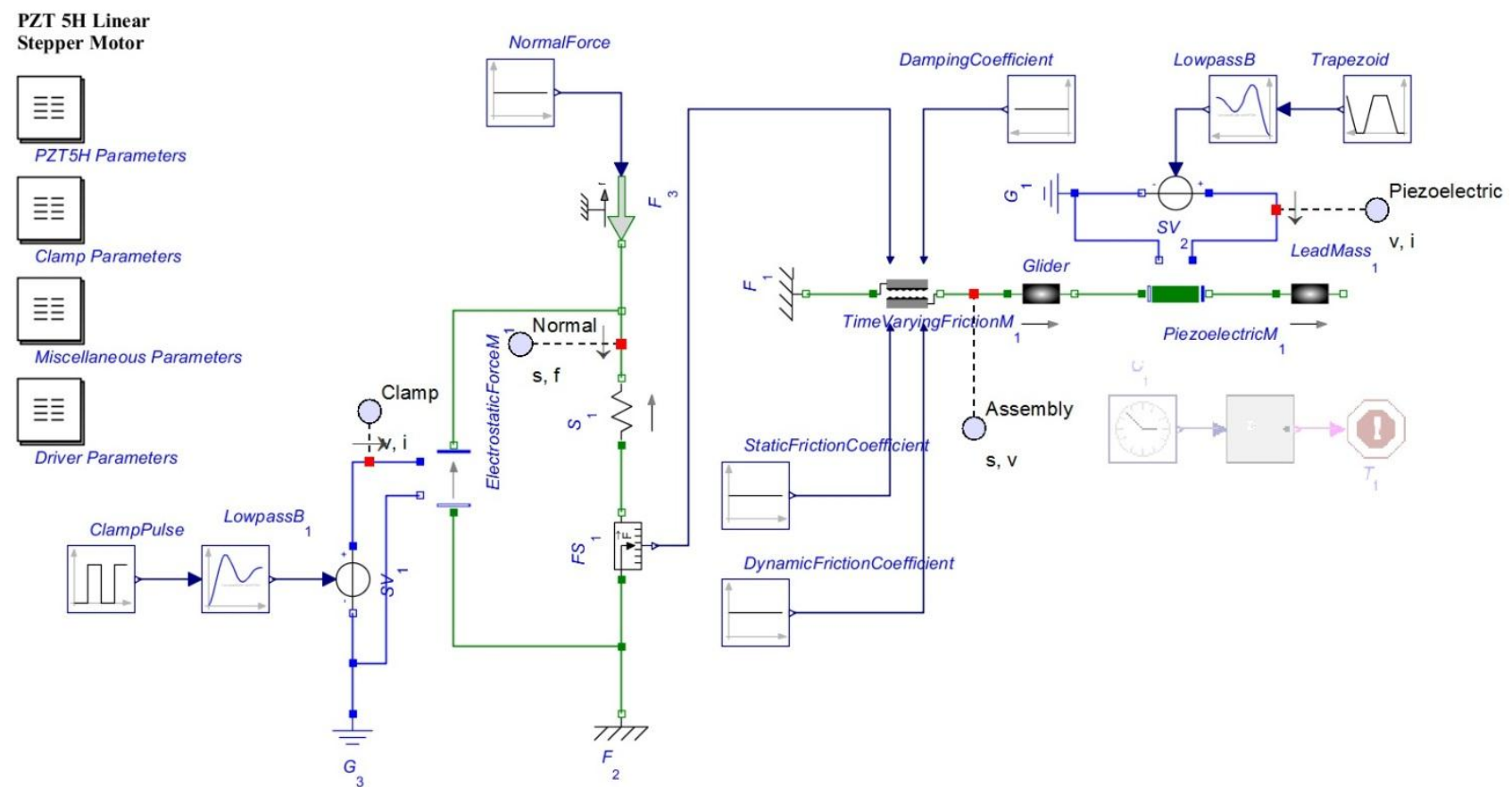

Figure 7: The MapleSim schematic of the parametrically-defined linear motor model.

The model was defined parametrically, using the parameters summarized in [2] as nominal values. Amazingly, the results matched quite well just by using the physical parameters and using some basic assumptions on the undocumented parameters, in particular, the characteristics of the driver waveforms. For example, it is stated in [2] that step sizes of $0.07-1.1 \mu \mathrm{m}$ were observed for piezoelectric voltages of $60-340 \mathrm{~V}$. The MapleSim model achieved $0.061-0.371 \mu \mathrm{m}$ step sizes for the same applied voltages without any tuning or optimization of the unknown parameters. Adjusting the magnitude of the clamp voltage and frequency cutoff of the filters are two of the easiest was of changing the step size to help it match the experimental results. Therefore, the MapleSim model represents a reasonable approximation to the system behavior without the burden of a full PDE solution.

\subsection{MapleSim Model and Preliminary Results}

As stated previously, the model matches the experimental results quite well and provides additional degrees of freedom to back-fit to the available data. Figure 8 and Figure 9 show the applied driver signals and resulting motor motion, respectively. A comparison to the results in [2] shows good qualitative and numerical agreement.

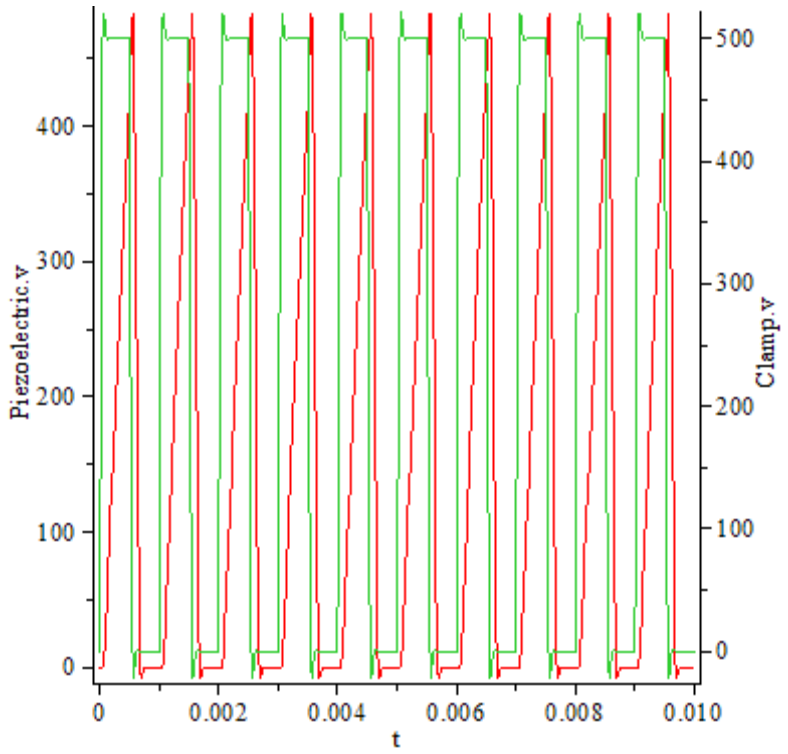

Figure 8: $1 \mathrm{kHz}$ clamp (green) and piezoelectric (red) drive voltage signals. The slight overshoot is due to a low-pass filter set to $10 \mathrm{kHz}$ to limit discontinuities present in the simulation. 


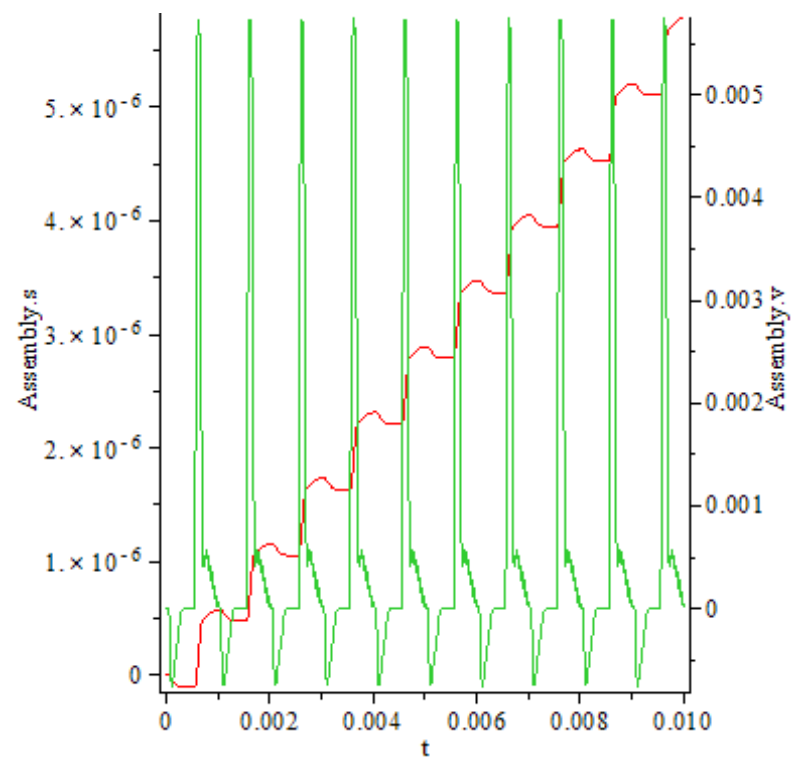

Figure 9: Plots of the position (red) and velocity (green) versus time of the linear motor.

\subsection{Platform for Optimization}

One of the goals of this research is to demonstrate the value of system-level models of devices that traditionally have only been modeled in PDE software. As an example of the execution speed and optimizations possible, consider the results in Figure 10, and further summarized in Figure 11. They show the position versus time and velocity versus $V_{\text {clamp }}$ results for 100 simulations, respectively. When comparing to the results presented in Fig. 10 in [2], it can be seen that the results are quite consistent.

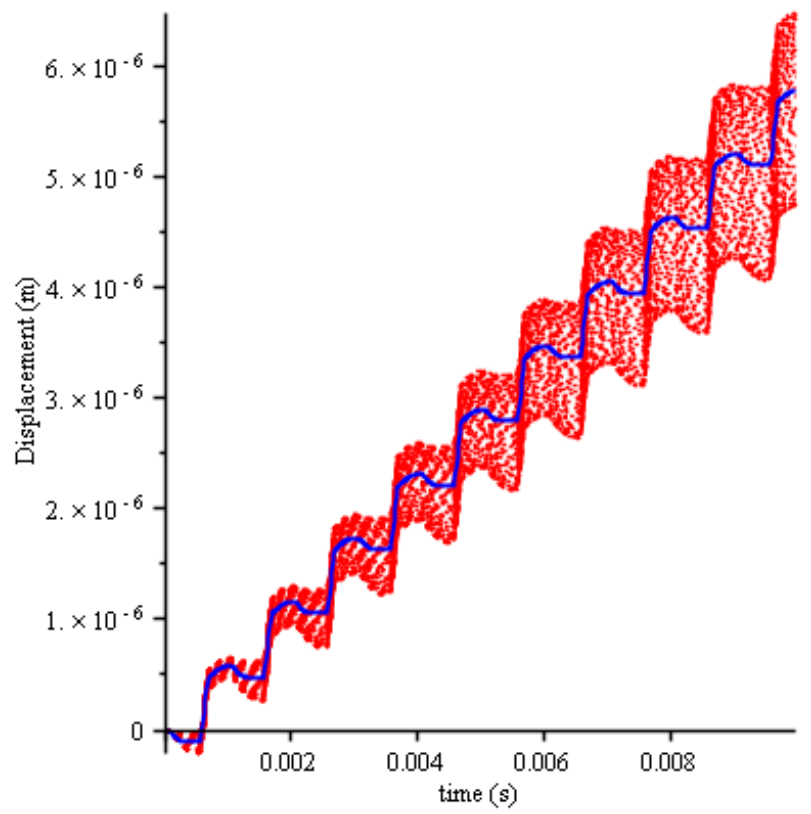

Figure 10: Position versus time plots for $V_{\text {clamp }}$ values from 0 to $1000 \mathrm{~V}$. The nominal value, $V_{\text {clamp }}=500 \mathrm{~V}$, is shown in blue.

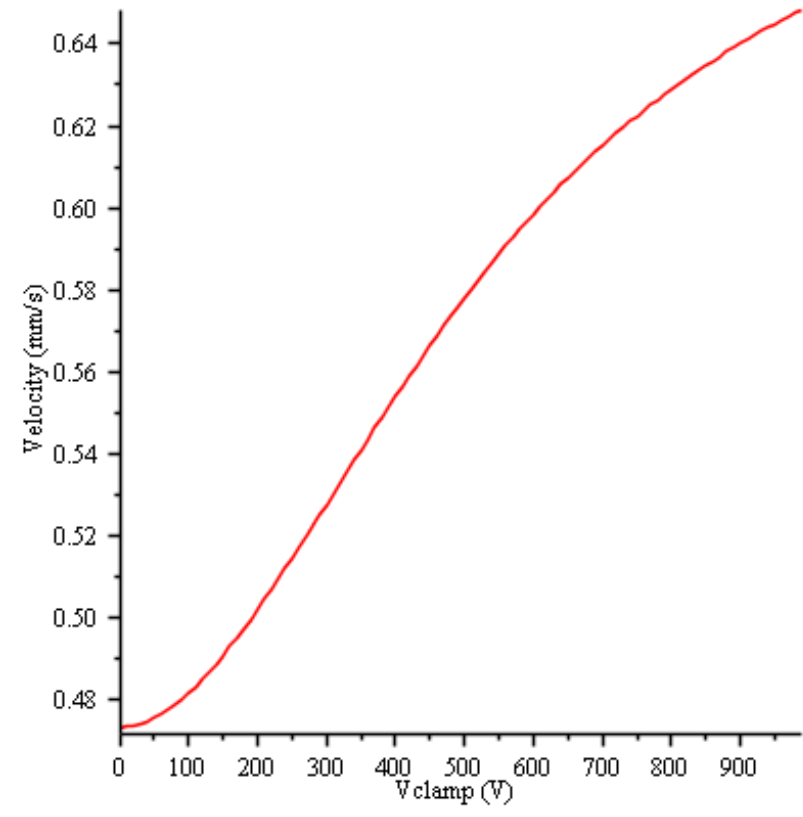

Figure 11: Calculated average velocity values for various $V_{\text {clamp. }}$. Note how the electrostatic clamp improves the speed of the motor by preventing reverse motion during extension of the piezoelectric material.

The per-simulation execution time was $63.8 \mathrm{~ms}$ on a modest Intel Core2 Duo CPU running at $2.80 \mathrm{GHz}$. Similar results would take a tremendous amount of time in PDE simulation software. Though the PDE results would arguably be more accurate, the marginal accuracy is of questionable value in light of the orders of magnitude increase in simulation time.

\section{Conclusions and Further Research}

This paper has demonstrated the creation of a linear piezoelectric stepper motor in MapleSim. To produce the motor model, three new components were created and their derivations were documented. Initial results correlate well with published experimental results, indicating that lumped-parameter system-level models may provide a new platform for development and optimization of such devices.

The follow-up research currently underway involves multi-parameter optimizations in a multithreaded, multicore architecture in Maple. The goal would be to demonstrate that fast MapleSim models can be used to optimize for goals like motor speed and power consumption, as well as to more accurately fit the experimental data. This will be accomplished directly in Maple via its threads and grid computing capabilities, and in Optimus, a global op- 
timization and design-of-experiments package by Noesis [8].

Using the piezoelectric material model as a starting point, further developments include a full multibody (6 DoF) model of the material behavior. It is created using the full tensor description of the piezoelectric material. This will enable the development of novel devices using torsional modes, and a more accurate look into the behavior of existing devices, like the motor presented in this paper.

\section{References}

[1] www.maplesim.com

[2] Judy J W, Polla D L, and Robbins W P. A Linear Piezoelectric Stepper Motor With Submicrometer Step Size and Centimeter Travel Range. IEEE Trans. UFFC, Vol. 37, No. 5, 1990.

[3] Schwarz P, and Schneider P. Model Library and Tool Support for MEMS Simulation. SPIE Proc. Microelectronic and MEMS Technology, Vol. 4407, 2001.

[4] Pecheux F, Allard B, Lallement C, Vachoux A, and Morel H. Modeling and Simulation of Multi-Discipline Systems Using Bond Graphs and VHDL-AMS. Proc. ICBGM, 2005.

[5] Gentili L, Bassi L, Macchelli A, Melchiorri C, and Borsari R. Model Reduction for HighOrder Port-Hamiltonian Systems. Application to Piezo-Electric Systems. Proc. IEEE Conf. Decision and Control, 2009.

[6] Essalam B A, and Mabrouk K. Generation of analytical redundancy relations for fault detection and isolation of ultrasonic linear motor. Nature \& Technology, Vol. 4, 2011.

[7] Cobbold R S C. Foundations of Biomedical Ultrasound (New York: Oxford University Press). 2007.

[8] www.noesissolutions.com 
